\title{
Avaliação do Programa de Tratamento do Tabagismo do Sistema Único de Saúde brasileiro em um munícipio do sudoeste de Minas Gerais
}

\author{
Evaluation of the Tobacco Treatment Program of the brazilian Unified Health System in a \\ municipality in the southwest of Minas Gerais
}

Evaluación del Programa de Tratamiento del Tabaquismo del Sistema Único de Salud brasileño en un municipio del suroeste de Minas Gerais

Recebido: 02/02/2021 | Revisado: 12/02/2021 | Aceito: 16/02/2021 | Publicado: 24/02/2021

Ciro Luiz Fernandes Reis ORCID: https://orcid.org/0000-0002-1039-0933 Universidade do Estado de Minas Gerais, Brasil E-mail: ciro.luiz11@gmail.com

Viviani de Oliveira

ORCID: https://orcid.org/0000-0003-3776-1498 Universidade do Estado de Minas Gerais, Brasil E-mail: viviani.2136949@discente.uemg.br Cristopher Mateus Carvalho ORCID: https://orcid.org/0000-0002-9778-1872 Universidade do Estado de Minas Gerais, Brasil E-mail: cristopher.matheus@hotmail.com

Leonardo Frasson Reis ORCID: https://orcid.org/0000-0003-4568-2220 Universidade do Estado de Minas Gerais, Brasil E-mail: leonardo.reis@uemg.br

Maria Inês Lemos Coelho Ribeiro ORCID: https://orcid.org/0000-0002-7684-2381 Universidade do Estado de Minas Gerais, Brasil E-mail: maria.ines@uemg.br

\begin{abstract}
Resumo
Introdução: Na década de 1980, surgiram as primeiras medidas contra o tabagismo pelo Ministério da Saúde brasileiro. A partir de 2002, o Programa de Tratamento do Tabagismo (PTT) foi implementado no Sistema Único de Saúde (SUS), fornecendo tratamento gratuito nas Estratégias de Saúde da Família (ESFs). Objetivos: Avaliar e descrever o PTT, aplicado em um município da região sudoeste de Minas Gerais, a partir de seus usuários, bem como traçar o perfil sociodemográfico desses indivíduos. Materiais e métodos: Trata-se de um estudo observacional, tipo transversal, com abordagem quantitativa e qualitativa, cuja coleta de dados ocorreu entre março de 2019 e março de 2020 por meio da aplicação de questionário sistematizado aos profissionais das ESFs alvo e a 52 usuários do PTT inscritos no programa. Resultados: Foi observado que a maioria dos pacientes pertencia ao sexo feminino e que $68 \%$ desses obtiveram sucesso na cessação do tabagismo após sua submissão ao PTT, sendo a terapia em grupo avaliada pelos usuários como o melhor tratamento. Foi apontada associação significativa $(p<0,05)$ entre o desfecho clínico e o tipo de tratamento empreendido, a quantidade de cigarros fumados por dia e o número de enfermidades. Segundo o relato dos profissionais, adaptações se fizeram necessárias segundo a realidade local do município com fins a melhorar os resultados do PTT. Conclusão: A pesquisa demonstrou que o PTT apresentou eficácia em promover a cessação do tabagismo. No entanto, mais estudos se fazem necessários com fins a avaliar a eficácia desse programa em outras realidades.
\end{abstract}

Palavras-chave: Tabagismo; Tratamento; Abandono do hábito de fumar; Sistema Único de Saúde; Atenção primária à saúde.

\begin{abstract}
Introduction: In the 1980s, the first measures against smoking were taken by the Brazilian Ministry of Health. As of 2002, the Smoking Treatment Program (STP) was implemented in the Unified Health System (UHS), providing free treatment at the Family Health Strategies (FHS). Objectives: To evaluate and describe the PTT, applied to its users in a city in the southwest region of Minas Gerais, as well as to outline the sociodemographic profile of these individuals. Materials and methods: This is an observational, cross-sectional study with a quantitative and qualitative approach, whose data collection took place between March 2019 and March 2020 through the application of a systematic questionnaire to the professionals of the target ESFs and 52 users of the PTT enrolled in the program. Results: It was
\end{abstract}


observed that most patients were female and that $68 \%$ were successful in smoking cessation after their submission to STP, with group therapy being evaluated by users as the best treatment. A significant association $(p<0.05)$ was found between the clinical outcome and the type of treatment undertaken, the number of cigarettes smoked per day and the number of illnesses. According to the professionals' reports, adaptations based on the local reality of the city were necessary in order to improve the results of the STP. Conclusion: Research has shown that STP was effective in promoting smoking cessation. However, more studies are needed in order to assess the effectiveness of this program in other realities.

Keywords: Tobacco use disorder; Treatment; Smoking cessation; Unified Health System; Primary health care.

\section{Resumen}

Introducción: En la década de 1980, aparecieron las primeras medidas contra el tabaquismo por parte del Ministerio de la Salud de Brasil. A partir de 2002, se implementó el Programa de Tratamiento del Tabaquismo (PTT) en el Sistema Único de Salud (SUS), brindando tratamiento gratuito en las Estrategias de Salud de la Familia (ESF). Objetivos: evaluar y describir la PTT, aplicada en un municipio de la región suroeste de Minas Gerais, desde sus usuarios, así como delinear el perfil sociodemográfico de estos individuos. Materiales y métodos: Se trata de un estudio observacional, transversal con abordaje cuantitativo y cualitativo, cuya recogida de datos se realizó entre marzo de 2019 y marzo de 2020 mediante la aplicación de un cuestionario sistemático a los profesionales de las ESF objetivo y 52 usuarios del PTT inscritos en el programa. Resultados: Se observó que la mayoría de los pacientes eran mujeres y que el $68 \%$ de estos lograron dejar de fumar después de su sumisión a PTT, siendo la terapia grupal evaluada por los usuarios como el mejor tratamiento. Se encontró una asociación significativa $(p<0,05)$ entre el resultado clínico y el tipo de tratamiento realizado, el número de cigarrillos fumados por día y el número de enfermedades. Según los informes de los profesionales, eran necesarias adaptaciones de acuerdo a la realidad local del municipio para mejorar los resultados del PTT. Conclusión: La investigación ha demostrado que el PTT fue eficaz para promover el abandono del hábito de fumar. Sin embargo, se necesitan más estudios para evaluar la efectividad de este programa en otras realidades.

Palabras clave: Tabaquismo; Tratamiento; Cese del hábito de fumar; Sistema Único de Salud; Atención primaria de salud.

\section{Introdução}

No final da década de 80, a existência de um sistema de saúde público no Brasil passou a ser uma realidade com a criação e a implementação do Sistema Único de Saúde (SUS). Esse foi instituído apresentando como seus princípios e diretrizes a integralidade, igualdade e universalidade de acesso em todos os níveis de assistência, participação da comunidade e descentralização político-administrativa (Viacava, Oliveira, Carvalho, Laguardia, \& Bellido, 2018).

A Atenção Primária à Saúde (APS) caracteriza-se por ser a principal porta de entrada do SUS. Nesse sentido, para que o usuário tenha acesso aos serviços do SUS em outros pontos de complexidade, como os de atenção secundária e terciária à saúde, ele é, geralmente, encaminhado a partir da Atenção Primária, ou seja, por uma Estratégia de Saúde da Família (ESF). Dessa maneira, a ESF possui importante função na organização das práticas da APS desde a prevenção à recuperação de doenças, promoção e reabilitação em saúde. Além disso, visa garantir cuidado continuado a partir da articulação entre atenção básica e demais pontos de complexidade da rede de saúde (Santos, Santos, \& Caccia-Bava, 2019).

Nesse contexto, a APS tem um papel fundamental na prevenção primária e secundária de doenças crônicas. O cuidado integral compreende o acompanhamento contínuo e esclarecimento acerca das doenças crônicas na população, que tem como um dos principais fatores de risco, o tabagismo. Esse provoca seis milhões de mortes a cada ano no mundo, sendo responsável por $71 \%$ das mortes por neoplasias pulmonares, $42 \%$ das doenças respiratórias crônicas e $10 \%$ das doenças cardiovasculares (Vargas, et al., 2017). No Brasil, 428 pessoas morrem por dia devido à dependência à nicotina (Brasil, 2019b).

Sabe-se que uma das estratégias para a evitar o câncer de pulmão, problema de saúde pública global e reduzir morbimortalidade de várias doenças crônicas é diminuir o consumo do cigarro (Souza, Cruz, \& Vasconcelos, 2016; Brasil, 2019b). Porém, conforme alertam Polosa, Caponnetto, Niaura e Abrams (2017), o hábito de fumar é um vício que traz consigo notória dificuldade para que haja sua superação. Dessa maneira, aqueles que fumam por muitos anos, costumam conviver com períodos cíclicos de remissão e recaída. As taxas de recaída, entre 40 e 70\%, após tratamento para cessação do vício, indicam uma clara necessidade de novos avanços para prevenir a reincidência (Souza, et al., 2019). 
Além da redução da qualidade de vida, o tabagismo em estágio avançado contribui para gastos financeiros com saúde pelo indivíduo e pelo Estado. No Brasil, os gastos gerados com o tratamento das doenças relacionadas ao tabaco e a perda de produtividade, resultante da morbidade e da mortalidade do tabaco, somaram custos de $\mathrm{R} \$ 21$ bilhões em 2011, considerando os sistemas de saúde públicos e suplementares (Mendes, et al., 2016).

A literatura demonstra que cerca de $80 \%$ dos fumantes desejam parar de fumar. No entanto, apenas 3\% dessa parcela conseguem a cada ano, e a maioria desse grupo cessa sem ajuda. Esse cenário se modifica quando programas de governo são implantados nas redes da APS, ampliando-se as taxas de cessação do tabagismo de 3\% para 20 a 30\% em um ano (Goyatá, Silva, Souza, Podestá, \& Beijo, 2014).

Na década de 1980, surgiram as primeiras medidas contra o tabagismo pelo Ministério da Saúde (MS). Hoje, a Política Nacional de Controle do Tabaco ações focalizadas, como promoção e apoio à cessação do tabagismo. A partir de 2002, o Programa de Tratamento do Tabagismo (PTT) foi implementado no SUS, com o objetivo de aumentar o acesso de indivíduos fumantes a ferramentas eficazes de cessação do vício, adotando-se métodos reconhecidos como primeira linha de tratamento (Mendes, et al., 2016).

O Programa Nacional de Controle do Tabagismo fornece tratamento gratuito pelo SUS nas Unidades Básicas de Saúde. As orientações sobre a organização do programa estão previstas nas diretrizes das portarias do MS no 571/2013 e 761/2016. As diretrizes de cuidado à pessoa tabagista expõem que todos os pontos da Rede de Atenção do SUS devem fornecer o tratamento adequado para o tabagismo, sobretudo nos serviços da APS (Brasil, 2013; Brasil, 2016).

Além disso, o Instituto Nacional de Câncer José Alencar Gomes da Silva (INCA) elabora um material, que é destinado aos coordenadores do programa, o "Manual do Coordenador - Deixando de Fumar sem Mistérios". Esse apresenta informações sobre o programa em relação ao perfil e papel do coordenador e às estruturas e conteúdo das sessões em grupo. Nesse manual, é informado que adaptações locais podem ser realizadas pelos coordenadores (Brasil, 2019a).

Os usuários do PTT recebem diferentes abordagens de tratamento que são definidas a partir de uma avaliação clínica inicial. O tipo de abordagem pode ser mínima ou intensiva, individual ou em grupo e, se necessário, pode-se lançar mão de terapia medicamentosa. Entretanto, tem-se que a intervenção-base é a terapêutica psicossocial. Essa última, no molde de um grupo operativo de apoio, é baseada em aconselhamento, materiais de autoajuda e abordagem cognitivo-comportamental. (Brasil, 2013; Brasil, 2015).

A portaria prevê que os grupos terapêuticos tenham entre 10 e 15 participantes e duração de 12 meses, no seguinte esquema: no primeiro mês são realizadas quatro sessões semanais, no segundo, duas sessões quinzenais e a partir do terceiro mês, sessões mensais para prevenção de recaídas, até completar um ano. As sessões devem ser coordenadas por dois profissionais de saúde de nível universitário, previamente capacitados e com habilidades de comunicação. (Brasil, 2016).

Essas e outras medidas contribuíram, em nível nacional, para a redução de 37,6\% do índice de fumantes de 2006 a 2018, de acordo com dados colhidos pelo Sistema de Vigilância de Fatores de Risco e Proteção para Doenças Crônicas por Inquérito Telefônico (Vigitel). Porém, em um ano, a proporção de adultos fumantes aumentou 0,5\% no país (Brasil, 2020a). Já em nível global, considerando, portanto, também as medidas implementadas por outros países, afirma-se que houve redução no índice de fumantes do sexo masculino, com 15 anos ou mais, de 43\% para 34\% entre os anos de 2000 e 2015. Em relação às mulheres, essa redução foi de 11\% em 2000 para 6\% em 2015 (World Health Organization [WHO], 2019).

Um ponto fundamental, que merece destaque no processo de cessação do tabagismo, é a terapia medicamentosa. Além do seu papel definido e reconhecido no processo de cessação do tabagismo, visa, principalmente, minimizar os sintomas da síndrome de abstinência da nicotina. Aliado a isso, outra questão que ganha destaque nesse processo se relaciona à intervenção psicossocial com abordagem cognitivo-comportamental, realizada nas ESFs (Brasil, 2015).

Diante do exposto e considerando a necessidade e importância de uma avaliação descentralizada da implementação do 
programa de cessação do tabagismo (instituído pelo MS) a nível municipal, este trabalho teve por objetivo descrever e avaliar o tratamento implementado para a cessação do tabagismo a partir da perspectiva dos usuários das ESFs de um município da região sudoeste de Minas Gerais, bem como mensurar os índices de sucesso na cessação do tabagismo e traçar o perfil sociodemográfico e epidemiológico desses usuários.

\section{Metodologia}

\subsection{Desenho do Estudo e Delineamento Amostral}

Baseado em Pereira, Shitsuka, Parreira e Shitsuka (2018), o presente trabalho foi delineado como um estudo observacional, tipo transversal, com abordagem qualitativa (método de investigação de base linguística-semiótica, com coleta de dados descritivos) e quantitativa (aquela na qual os resultados podem ser quantificados). O delineamento amostral adotado foi o não probabilístico.

Os participantes do estudo foram 52 usuários do PTT de um município da região sudoeste de Minas Gerais, maiores de 18 anos e inscritos no programa.

A pesquisa foi executada no período compreendido entre março de 2019 e março de 2020 nas ESFs do municípiosede desse estudo durante as reuniões de terapia em grupo do programa e nas residências dos usuários que atendessem aos prérequisitos para serem incluídos na pesquisa e aceitassem, por assinatura, as condições do Termo de Consentimento Livre e Esclarecido (TCLE).

Foram realizadas entrevistas individuais com os usuários presentes, deixando-os livres para expressar sua opinião acerca do programa, apontando aspectos positivos e limitações do mesmo. Em seguida, era aplicado questionário sistematizado de elaboração própria para coleta de dados, coletando-se os seguintes itens:

a) Informações sociodemográficas: idade, sexo, etnia, estado civil, escolaridade, renda total dos integrantes da residência (renda familiar) e exercício de ocupação remunerada.

b) Presença de comorbidades: doenças cardiovasculares, diabetes, hipertensão, dislipidemia, doenças no fígado, nos rins, doenças intestinais, problemas respiratórios, osteoporose, câncer.

c) História tabagística do indivíduo: tempo de cessação e tempo total de tabagismo, idade de início do tabagismo, quantidade de cigarros fumados por dia e número de tentativas anteriores de cessação.

d) Avaliação do Programa e da(s) Terapia(s) utilizada(s): tipos de tratamento utilizados através do PTT, avaliação desses tratamentos e nível de satisfação dos usuários em relação ao PTT.

Os dados dos usuários que tiveram evasão do PTT foram solicitados às coordenadoras do programa no município. Assim, executou-se busca ativa dos inscritos com o objetivo de aumentar o alcance dos usuários.

Além disso, foram realizadas entrevistas orais com duas coordenadoras municipais do programa e com os profissionais da equipe que conduzem as reuniões para obter informações sobre o funcionamento e arranjo do programa no município. As perguntas eram abertas, tais como "Como é a organização do programa no município?", "Qual é o tempo de duração do programa em cada ESF?", “Como são conduzidas as sessões de terapia em grupo?”, entre outras.

\subsection{Análise dos Dados}

Os dados coletados pelos instrumentos foram armazenados em uma planilha eletrônica e analisados no software IBM SPSS® 23. Foi realizada a análise exploratória de dados, obtendo-se a estatística descritiva da população estudada. Para inferências e análises de associação entre as variáveis de interesse e a variável dependente (sucesso na cessação do tabagismo), empregou-se o teste qui-quadrado $\left(\chi^{2}\right)$ adotando-se um erro de até $5 \%(\mathrm{p}<0,05)$.

As variáveis independentes foram: idade, estado civil, etnia, atividade remunerada, escolaridade e renda, presença de 
enfermidades, e a história tabagística, representada pelos itens: idade de início do tabagismo, quantidade de cigarros fumados por dia, tempo total de tabagismo e tipo de tratamento empreendido.

A variável dependente foi o índice de sucesso a curto prazo, obtido ao final do tratamento. Para tanto, foi considerado como critério para status de sucesso na cessação à curto prazo, o tempo mínimo de 1 mês sem fumar.

Para identificar a influência dos fatores associados ao desfecho da variável dependente, foi empregado um algoritmo de regressão logística binomial. Na elaboração deste modelo, as variáveis independentes que apresentaram p $>0,10$ foram usados como variáveis de entrada. Como estimativa de força de associação foi utilizado a razão de chances, intervalo de confiança (IC 95\%). Foi adotada como significância no modelo final p <0,05.

\subsection{Aspectos Éticos}

O presente estudo foi aprovado pelo Comitê de Ética em Pesquisa da Universidade do Estado de Minas Gerais (UEMG), por meio da Plataforma Brasil (número CAAE 13806719.9.0000.5112).

\section{Resultados}

\subsection{Perfil Sociodemográfico e Avaliação do Programa segundo Usuários}

Foram entrevistados 52 participantes, com idade média de 54,48 anos (DP: $\pm 10,46$ ) (idade mín.: 23 e máx.: 71 anos). Houve predominância do sexo feminino $(63,5 \%, \mathrm{n}=33)$ e com idade maior ou igual a 60 anos $(36,5 \%, \mathrm{n}=19)$. Além disso, os participantes, em sua maioria, possuíam primeiro grau completo como nível de escolaridade $(42,3 \%, \mathrm{n}=22)$ e eram, em sua maioria, casados $(63,5 \%, n=33)$. Dos entrevistados, 63,4\% $(n=33)$ não exerciam atividade remunerada. Os aspectos sociodemográficos estão demonstrados na Tabela 1.

Tabela 1. Aspectos sociodemográficos da amostra.

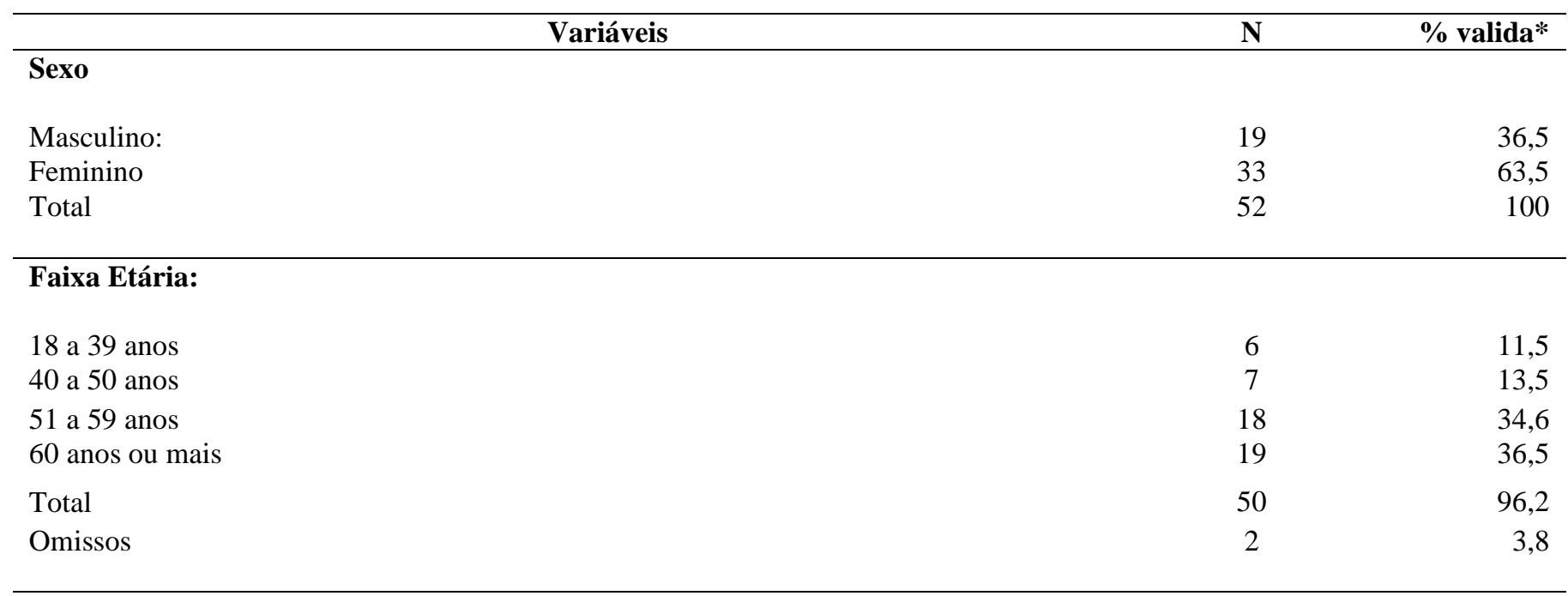

\section{Estado Civil}

Solteiro

Casado

Viúvo

Divorciado

Total

Omissos 


\section{Escolaridade}

Analfabeto / Fundamental Incompleto

Fundamental I** completo

17,3

Fundamental II*** completo

Ensino médio completo

Ensino superior completo

Total

11,5

Total

Omissos

\section{Renda Familiar}

Até 1,5 salários mínimos

De 1,5 a 3 salários mínimos

De 4 a 7 salários mínimos

Total

Omissos

\section{Comorbidades}

Por quantidade de comorbidades

Possui uma comorbidade

Possui duas comorbidades

Possui três ou mais comorbidades

Total

Não possui

Por tipo de comorbidade

Hipertensão

Dislipdemia

Doenças cardíacas

Diabetes

Doenças respiratórias

Osteoporose

Hepatopatias

Doenças intestinais

*Porcentagem válida desconsidera casos omissos. **Fundamental $\mathrm{I}=1^{\circ}$ ao $5^{\circ}$ ano. $* * *$ Fundamental $\mathrm{II}=6^{\circ}$ ao $9^{\circ}$ ano. Fonte: Autoria própria.

No que se refere à história tabagística, a estatística descritiva referente à esse tópico está demonstrada na Tabela 2. 
Tabela 2. Distribuição dos usuários segundo aspectos de sua história tabagística.

\begin{tabular}{|c|c|c|c|}
\hline & Variáveis & $\mathbf{N}$ & \% válida* \\
\hline \multicolumn{4}{|c|}{ Idade de início do tabagismo } \\
\hline 6 a 10 anos & & 6 & 11,5 \\
\hline 11 a 17 anos & & 28 & 53,8 \\
\hline 18 a 25 anos & & 13 & 25 \\
\hline 26 a 35 anos & & 2 & 3,8 \\
\hline 51 anos ou mais & & 1 & 1,9 \\
\hline Total & & 50 & 96,2 \\
\hline Omissos & & 2 & 3,8 \\
\hline \multicolumn{4}{|c|}{ Tempo total de tabagismo } \\
\hline Menos de 5 anos & & 3 & 5,8 \\
\hline 5 a 10 anos & & 2 & 3,8 \\
\hline 11 a 20 anos & & 3 & 5,8 \\
\hline 21 a 30 anos & & 4 & 7,7 \\
\hline 31 anos ou mais & & 39 & 75 \\
\hline Total & & 51 & 98,1 \\
\hline Omissos & & 1 & 1,9 \\
\hline
\end{tabular}

\section{Quantidade de cigarros/dia}

1 a 5

6 a 15 cigarros

Mais de 30

Total

Omissos

\section{Desistências em tratamentos anteriores}

Nenhuma

$1 \mathrm{vez}$

-

\footnotetext{
* Porcentagem válida desconsidera casos omissos. Fonte: Autoria própria.
}

No que tange ao tipo de tratamento e a sua avaliação segundo a percepção dos usuários, tem-se que todos os indivíduos pesquisados $(\mathrm{n}=52)$ participavam da terapia em grupo e a tinham como melhor método empreendido. O tipo de tratamento utilizado via programa, incluindo os medicamentos, como o adesivo transdérmico de nicotina (ATN) e o cloridrato de bupropiona (bupropiona), bem como a avaliação desses, estão explanados na Tabela 3. 
Tabela 3. Tipo de tratamento utilizado e avaliação segundo a percepção dos usuários.

\section{Tratamentos utilizados}

Terapia em Grupo, sendo que:

Sem tratamento medicamentoso

Somente ATN

Somente Bupropiona

ATN + Bupropiona

51 respostas, 1 caso omisso

\section{Avaliação da Terapia em Grupo}

Péssimo

Ruim

Regular

Bom

Muito bom

48 respostas, 4 casos omissos

2,1

2,1

2,1

$\begin{array}{rr}5 & 10,4 \\ 40 & 83,3\end{array}$

\section{Avaliação do ATN}

Péssimo

Ruim

Regular

Bom

Muito bom

40 respostas, 12 casos omissos

\section{Avaliação do Bupropiona}

Péssimo

Ruim

Regular

Bom

Muito bom

29 respostas, 23 casos omissos

*Porcentagem válida desconsidera casos omissos. Fonte: Autoria própria.

Quando se trata do nível de satisfação geral dos usuários em relação ao programa, 71,2\% (n=37) escolheram a opção "muito satisfeito", 21,1\% (n=11) "satisfeito" e 3,8\% (n=2) "pouco satisfeito". Outros 3,8\% (n=2) assinalaram a opção "totalmente insatisfeito".

Conforme já elucidado na metodologia, esse estudo considerou como sucesso a curto prazo, o usuário que declarou estar há pelo menos 1 mês sem o uso do tabaco. Ao final do estudo, 68\% (n=35) dos participantes declararam cessação do vício, sendo que 26,9\% ( $n=14)$ haviam parado de fumar há menos de um mês e 40,4\% $(n=21)$, entre um e seis meses. Apenas um paciente, que representa $1,9 \%$ da amostra, relatou ter parado há mais de um ano. Portanto, pelo critério adotado, considerou-se que $42,3 \%$ alcançaram sucesso na cessação do vício à curto prazo.

Desse modo, a Tabela 4 demonstra os dados referentes a terapêutica empreendida relacionados com o desfecho alcançado pelos indivíduos. 
Tabela 4. Relação entre o tipo de tratamento utilizado e o desfecho clínico do usuário.

\begin{tabular}{lccc}
\hline \multicolumn{1}{c}{ Tratamento } & Sucesso mais de 1 mês & \\
\cline { 2 - 4 } & Não parou de fumar & Parou de fumar & Total \\
\hline Terapia em grupo (TG) & 6 & 7 & 13 \\
Adesivo transdérmico (AT) & 1 & 4 & 5 \\
Bupropiona & 3 & 2 & 0 \\
TG + AT & 1 & 1 & 1 \\
TG + Bupropiona & 0 & 1 \\
TG + AT + Bupropiona & 1 & 1 \\
AT + Bupropiona & 0 & 1 \\
TG + AT + GM / P + Bupropiona & 0 & 1 \\
Nenhum & 0 & 1 \\
Não parou de fumar & 16 & 1 \\
Não sabe & 1 & 2 \\
Total & 29 & 1 \\
\hline
\end{tabular}

Fonte: Autoria própria.

As avaliações estatísticas obtidas através dos testes de Qui-quadrado $\left(\chi^{2}\right)$ apontaram associação estatisticamente significativa $(\mathrm{p}<0,05)$ do sucesso na cessação do tabagismo com o tipo de tratamento empreendido, o número de cigarros fumados por dia e a presença de enfermidades, com valores de $\chi^{2}$ de 8,983; 11,074 e 22,5 e V de Cramer igual a 0,424; 0,471 e 0,67, respectivamente. Outras variáveis de interesse pesquisadas não demonstraram diferenças significativas $(\mathrm{p}>0,05)$.

Ainda em relação à avaliação estatística, a Tabela 5 apresenta o resultado da regressão logística, com os respectivos graus de significância, razão de chances e os intervalos de confiança (95\%). 
Tabela 5. Análise de regressão logística binomial para associação do número de enfermidades com o sucesso na cessação do tabagismo dos participantes.

\begin{tabular}{lcccc}
\hline & & \multicolumn{2}{c}{ Intervalo de confiança 95\% } \\
\cline { 2 - 5 } Variável preditora & $\mathbf{p}$ & Razão de chance & Inferior & Superior \\
\hline Intercepto & 0.038 & 0.200 & 0.0438 & 0.913 \\
N. enfermidades: & & & 2.4623 & 107.242 \\
$\quad$ Uma enfermidade - Nenhuma enfermidade & 0.004 & 16.250 & 1.2801 & 78.117 \\
$\quad \begin{array}{l}\text { Duas enfermidades - Nenhuma enfermidade } \\
\text { Três enfermidades - Nenhuma enfermidade }\end{array}$ & 0.028 & 10.000 & 0.7906 & 1.3908 \\
Cinco enfermidades - Nenhuma enfermidade & 0.081 & 6.667 & 20.000 & 287.600 \\
\hline
\end{tabular}

As estimativas representam as chances de log de "SucessoCessaçãoMaisDe1Mês = Não parou de fumar" vs. "SucessoCessaçãoMaisDe1Mês $=$ Parou de fumar". Fonte: Autoria própria.

\subsection{Descrição da Organização do Programa no Município}

Foram realizadas adaptações no formato do programa no município em relação às características previstas nas diretrizes das portarias do MS n ${ }^{\circ}$ 571/2013 e $\mathrm{n}^{\circ}$ 761/2016. (Brasil, 2013; Brasil, 2016). O Quadro 1 compara diversos aspectos da organização do programa adotada no município com as diretrizes dessas portarias.

Outros aspectos do programa, como: número de coordenadores por reunião, duração e temas das reuniões, presença de convidados palestrantes, estilo de abordagem em grupo, e entrega de materiais de apoio são reproduzidos de forma fiel à diretriz. 
Quadro 1. Comparativo entre a organização do programa de acordo com as diretrizes presentes nas portarias e organização adotada no município.

\begin{tabular}{|c|c|c|}
\hline Tópicos & $\begin{array}{c}\text { Organização do PTT de acordo } \\
\text { com as portarias }\end{array}$ & $\begin{array}{c}\text { Organização do PTT no } \\
\text { município }\end{array}$ \\
\hline $\begin{array}{l}\text { Duração total dos grupos e } \\
\text { periodicidade das reuniões } \\
\text { em grupo }\end{array}$ & $\begin{array}{c}\text { Duração total dos grupos: } 12 \\
\text { meses } \\
\text { Primeiro mês: reuniões semanais } \\
\text { Segundo mês: reuniões quinzenais } \\
\text { Terceiro mês em diante: reuniões } \\
\text { mensais }\end{array}$ & $\begin{array}{l}\text { Duração total dos grupos: } 3 \\
\text { meses. } \\
\text { Reuniões semanais. }\end{array}$ \\
\hline Oferta do programa & $\begin{array}{l}\text { Contínua durante o ano todo nas } \\
\text { ESFs credenciadas como } \\
\text { ofertantes do programa }\end{array}$ & $\begin{array}{c}\text { Rotativa* e temporária } \\
\text { (semestralmente, com duração } \\
\text { de } 3 \text { meses) entre as ESFs } \\
\text { credenciadas como ofertantes do } \\
\text { programa. }\end{array}$ \\
\hline $\begin{array}{c}\text { Condições para os usuários } \\
\text { receberem terapia } \\
\text { medicamentosa }\end{array}$ & $\begin{array}{c}\text { Admissão em terapia } \\
\text { medicamentosa na avaliação } \\
\text { inicial, de acordo com as } \\
\text { condições estipuladas pela } \\
\text { portaria do MS n }{ }^{\circ} 761 \text { de } 21 \text { de } \\
\text { junho de } 2016 .\end{array}$ & $\begin{array}{c}\text { Além da inclusão em terapia } \\
\text { medicamentosa de de acordo } \\
\text { com as condições da portaria, é } \\
\text { necessário o comparecimento às } \\
\text { reuniões semanais de terapia em } \\
\text { grupo para retirada dos } \\
\text { medicamentos }\end{array}$ \\
\hline $\begin{array}{l}\text { Medicamentos disponíveis } \\
\text { utilizados para cessação do } \\
\text { tabagismo }\end{array}$ & $\begin{array}{l}\text { Adesivo transdérmico de } \\
\text { nicotina (7 mg, } 14 \mathrm{mg} \text { e } \\
21 \mathrm{mg}) ; \\
\text { - Cloridrato de bupropiona, } \\
\text { comprimido (150mg) } \\
\text { Goma de mascar e } \\
\text { pastilha (ambos } 2 \mathrm{mg})\end{array}$ & 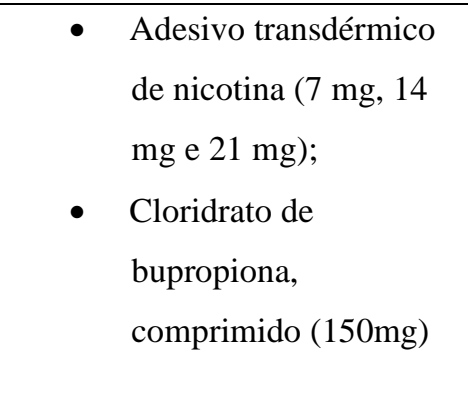 \\
\hline
\end{tabular}

Fonte: Autoria própria.

*Oferta rotativa do programa = a cada semestre, cerca de seis ESFs das existentes no município são aleatoriamente escolhidas pela coordenação para ofertar o Programa. Essa escolha, porém, deve, obrigatoriamente, contemplar ESFs matriciadadas por cada um dos três Núcleos de Apoio à Saúde da Família do município de forma equilibrada. No semestre consecutivo, outras ESFs são escolhidas para ofertá-lo e assim por diante. Outra questão importante a ser levada em consideração é que somente os usuários cadastrados nas ESFs ofertantes podem participar do programa em vigência naquele período/semestre.

Durante a fase de entrevistas com os profissionais, duas orientadoras dos Núcleos de Saúde da Família relataram que as alterações na periodicidade das reuniões e tempo total do programa foram realizadas como resposta a um problema de baixa adesão no município. 
Na implementação do programa, foi preconizado que os grupos teriam duração de um ano como previsto pelo MS. Entretanto, através dessa experiência, foi observado que reuniões com intervalos maiores que quinze dias resultavam em menor adesão e maior evasão de participantes. Diante disso, testaram-se novos esquemas de periodicidade para os grupos. Alguns desses incluíam uma duração total dos grupos de seis meses e outro de três meses, sendo que no primeiro eram realizados cinco encontros semanais, três quinzenais e o restante mensal e no segundo, eram realizadas reuniões semanais. Segundo as coordenadoras, a duração total dos grupos de três meses com reuniões semanais apresentou melhores resultados, aumentando-se a adesão dos usuários.

\section{Discussão}

Este estudo descreveu e avaliou o tratamento implementado para a cessação do tabagismo nas ESFs de um município da região sudoeste de Minas Gerais com o intuito de apontar lacunas a serem preenchidas e promover a melhoria da implementação do programa de cessação do tabagismo do MS a nível municipal. Além disso, forneceu um exemplo com o qual outros municípios podem efetuar comparações de suas experiências e a partir disso implementar medidas para mudanças que, porventura, se façam necessárias.

Com relação ao perfil amostral, observou-se uma maior participação do sexo feminino no PTT, o que corrobora com os dados publicados pelo MS no ano de 2020 (Brasil, 2020b). Estes demonstraram uma tendência constante da maior prevalência do sexo feminino em relação ao masculino na procura pelos serviços de saúde, notadamente com fins a alcançar tratamento para a cessação do tabagismo.

De acordo com Costa-Júnior, Couto e Maia (2016), a diferença entre os sexos, no que diz respeito à taxa de procura pelos serviços de saúde, ocorre devido a marcas culturais e sociais da concepção do gênero. Nesse viés, no que diz respeito ao sexo masculino, para muitos homens, a demonstração de fragilidade ou adoecimento físico produz consequências negativas, que podem acarretar em desmerecimento social. Já em relação as mulheres, tem-se que essas são ensinadas a cuidar da sua saúde desde cedo, devido, por exemplo, à concepção social de que o corpo feminino é frágil e mais predisposto ao adoecimento.

Tal contraste entre os sexos, sinaliza a necessidade da implementação de medidas que visem o maior alcance da população masculina pelos serviços de saúde, sobretudo no que se refere a cessação do uso do tabaco. Além disso, somando aos fatos que motivam a organização e aplicação de ações voltadas para os homens, vale ressaltar que a prevalência do uso do cigarro na população masculina é superior à da população feminina, fato que foi apontado por Bazotti, Finokiet, Conti, França e Waquil (2016) em seus estudos e também pelo MS com base em dados do Vigitel do ano de 2019 (Brasil, 2020a).

Nesse cenário, dentre as medidas passíveis de serem tomadas, ganham destaque as ações de educação em saúde voltadas para a população masculina. Essas ações, dentre outras caraterísticas, em seu planejamento e ao serem implementadas devem levar em consideração toda complexidade sociocultural que norteiam a atenção a saúde aos indivíduos do sexo masculino. Além disso, também devem lançar mão dos mais variados meios de comunicação e tecnologia da informação, assim como destacado por Pinto, Scopacasa, Bezerra, Pedrosa e Pinheiro (2017) e Carlotto e Dinis (2018), de maneira a atuar de forma efetiva na promoção da saúde masculina a partir da quebra de tabus/estigmas e ampliação da procura/acesso aos serviços de saúde.

No que se refere à idade, tem-se que a população que buscou o programa era majoritariamente composta por pessoas com idade maior que 60 anos $(36,5 \%)$ e que já possuíam ao menos uma enfermidade $(78,84 \%)$ com destaque para a hipertensão e a dislipidemia. Tal fato corrobora com a realidade de saúde nacional, uma vez que é sabido que com o avançar da idade há maior surgimento de comorbidades como as doenças crônicas e, portanto, maior procura pelos serviços de saúde. 
Além disso, mesmo em idades avançadas, vários benefícios podem ser obtidos com a cessação do tabagismo o que motiva ainda mais a procura por programas que visam a cura/controle desse vício (Santos et al., 2019).

No que tange ao estado civil dos participantes, destaca-se grande proporção de indivíduos casados, o que pode ser tanto positivo, quanto negativo no que se refere ao sucesso no fim do uso do tabaco. Nesse sentido, tem-se que o apoio social influencia de forma significativa na decisão do tabagista abandonar o vício e procurar ajuda. Diante disso, a presença de uma pessoa tabagista não colaborativa, por exemplo, morando junto no mesmo ambiente que um indivíduo sob tratamento para cessação do uso do tabaco pode influenciar de forma negativa o desfecho do parceiro e dificultar ainda mais o tratamento (França et al., 2015; Aragão, Portugal, Campos, Lopes, \& Fortes, 2017).

Assim, ações que visam o entendimento e envolvimento de toda rede familiar e social de um determinado indivíduo sob tratamento representam um importante passo com fins a melhorar os resultados do PTT.

Os achados sociodemográficos desse estudo sugerem baixa escolaridade e predominância de classes socioeconômicas desfavorecidas, sendo congruente com a literatura que demonstra uma associação entre menores níveis de escolaridade e renda com maiores prevalências do tabagismo (Barbosa \& Machado, 2015; Bazotti et al., 2016).

Indivíduos que apresentam elevado nível de dependência do cigarro, medido pelo Teste de Fagerström, necessitam de tratamento medicamentoso, assim como previsto pelo PTT proposto pelo MS (Brasil, 2016). No entanto, o tratamento validado e preconizado para o tabagismo na literatura médica pode representar um desafio em termos financeiros para os indivíduos que dele necessitam conforme já evidenciado por Mendes et al. (2016).

Isso salienta a importância do PTT na APS do SUS para as classes socioeconômicas menos favorecidas e endossa ainda mais a necessidade de medidas com fins a ampliação do alcance e escopo do programa para as mais diversas realidades brasileiras. Programas e medidas dessa natureza apresentam potencial para contribuir de maneira significativa no que tange a redução da prevalência do tabagismo no Brasil. Isso é ainda mais fortificado quando se considera que $84 \%$ da população tabagista recebe entre um e três salários mínimos per capita e, portanto, os indivíduos a ela pertencentes muitas vezes não têm condições de arcar por si só com os tratamentos que se fazem necessários (Bazotti et al., 2016).

Ressalta-se, no entanto, que a disponibilização de medicamentos no município por meio do PTT cessa após três meses. Após esse período, para receberem mais exemplares, os usuários devem esperar que o programa seja ofertado novamente em suas ESFs de referência para que então possam se reinscreverem.

Assim, fica evidente a necessidade de se ampliar o tempo de oferta dos benefícios do PTT, adicionando sessões de manutenção ou, se isso não for possível, mantendo a disponibilização medicamentosa durante um período maior para pacientes com elevada dependência à nicotina e para os quais 3 meses não será suficiente para que se alcance êxito no tratamento.

Nesse interim, a Sociedade Brasileira de Pneumologia e Tisiologia, por exemplo, em sua diretriz para cessação do tabagismo, sugere que o paciente em processo de cessação seja acompanhado por pelo menos seis meses subsequentes (Reichert, 2008 apud França et al., 2015).

Quanto a idade de início do tabagismo, observou-se que essa estava principalmente concentrada nas faixas etárias menores que 25 anos (90,3\%). Um padrão que também foi observado em vários outros estudos (Azevedo et al., 2009; Meier, Vannuchi, \& Oliveira, 2012; Goyatá et al., 2014) e que deve ser levado em consideração nas campanhas educativas e nas políticas públicas nacionais com fins ao controle do uso do tabaco.

Dentre as medidas passíveis de serem tomadas para mudança desse cenário, destaca-se a possibilidade de profissionais e acadêmicos de cursos superiores executarem atividades de promoção da saúde e prevenção de danos e agravos relacionados ao tabaco voltadas para o público jovem das escolas e universidades. Além disso, a divulgação de conteúdos educativos em mídias sociais, tais como o Instagram, e programas de rádio/televisão também poderia exercer um significativo 
impacto no que diz respeito à conscientização da população jovem e a ampliação da efetividade e escopo das ações da APS (Santos et al., 2019).

No que se refere à porcentagem de sucesso na cessação do tabagismo, levando-se em conta o critério do tempo mínimo de 1 mês sem fumar, 42,3\% dos participantes obtiveram êxito em sua empreitada.

Ao comparar essa taxa de sucesso com outros estudos que também avaliaram programas de intervenção para o tabagismo, observa-se que ela é um pouco inferior. Meier et al. (2012), por exemplo, analisou a eficácia de um programa de combate ao tabagismo por meio de 92 pacientes submetidos a um ano de tratamento e constatou que $66,6 \%$ desses atingiram o sucesso. Lucchese, Vargas, Teodoro, Santana, e Santana (2013), por sua vez, acompanhando o tratamento de 20 pessoas, também por um ano, observou uma taxa de sucesso de $78 \%$.

Tal fato pode ser justificado em partes pelo tempo de tratamento ao qual os participantes desse estudo eram submetidos, a questões de acesso aos serviços de saúde do município em questão e a adesão/continuidade do tratamento pelos pacientes. Dessa forma, fica evidente a necessidade da implementação de medidas com fins a mudança desse cenário. Dentre elas, a busca ativa por indivíduos em uso do tabaco e o acompanhamento domiciliar daqueles que já se encontram em tratamento ganham importante destaque quando se fala em melhoria de acesso e adesão/continuidade de tratamentos.

Em relação a avaliação estatística, a regressão logística apontou que a presença de alguma enfermidade aumenta as chances de sucesso na cessação do tabagismo a curto prazo, sendo a razão de chances mais elevada encontrada nos indivíduos com cinco ou mais enfermidades $(\mathrm{RC}=20)$.

No que tange a esse achado, a literatura traz que as informações quanto aos malefícios do consumo de cigarro para a saúde são difundidas mundialmente, mas só esse fato parece insuficiente para levar as pessoas a pararem de fumar. No entanto, percebe-se que o conhecimento sobre os agravos à saúde que o tabaco acarreta e a presença desses males auxilia o fumante a observar e a relacionar suas disfunções corporais/mentais ao seu vício (Lucchese, et al., 2013).

Em um estudo que avaliou os usuários de um programa similar ao PTT em Goiás, cujos achados também corroboram com os encontrados pela presente pesquisa, foi observado que os principais motivos que levaram os indivíduos a desejar parar de fumar foram a preservação e a restauração da saúde. Esse estudo identificou que a motivação pela busca da melhoria do estado físico ou psíquico ocorreu após alguma alteração no estado geral ou a vivência de uma situação real de acometimento à saúde (Lucchese et al., 2013).

Dessa forma, fica evidente que a procura por tratamento para a cessação do tabagismo ocorre quando os indivíduos fumantes começam a sentir os efeitos desse ato em sua saúde. Assim, vê-se a necessidade de uma maior intensificação das campanhas de combate ao uso do tabaco em fases que antecedem o aparecimento/complicação de comorbidades. Essa intensificação deve envolver a indústria do tabaco e ter um enfoque maior nos prejuízos que esse vício pode acarretar.

Ainda com relação a avaliação estatística, o teste do qui-quadrado apontou que a quantidade de cigarros fumados ao dia tem influência no sucesso do tratamento, com uma associação entre esses fatores ( $p<0,05, V$ de Cramer $=0,42)$. Esse achado é consistente com a literatura, que cita como medidas específicas de gravidade da dependência, o número de cigarros fumados por dia (DPC) e o tempo até o primeiro cigarro do dia. Esses fatores têm se mostrado consistentes na previsão do sucesso da manutenção da cessação do tabagismo (Higgins et al., 2018).

Ademais, é conhecido que a nicotina é o constituinte responsável pela dependência química do tabaco. Quanto mais alto o número de cigarros por dia, maior o nível sérico dessa substância e maior a dependência. Estudos mostram que a redução do teor dessa substância nos cigarros acarreta em reduções significativas no número de cigarros diários, redução da dependência de nicotina e aumentos nas tentativas de parar, com pouca evidência de tabagismo compensatório (Donny et al., 2015). 
No que tange ao tipo de tratamento e o desfecho de sucesso na cessação do tabagismo, a avaliação estatística revelou associação significativa entre essas duas variáveis, sendo a terapia de grupo o principal tratamento associado à taxa de sucesso. Esse achado vai de encontro aos já conhecidos desfechos e benefícios apontados por esse tipo de terapia por diversos estudos, tais como o de Santana, Vasconcellos e Ribeiro (2017) e de Paiva et al. (2017), e sinaliza a importância da promoção e intensificação desse tipo de terapia na APS com fins a cessação do tabagismo.

Quanto à organização do PTT no município-sede desse estudo, e levando em consideração os resultados obtidos por essa pesquisa, tem-se que adaptações locais na oferta do programa se fizeram necessárias, considerando-se a realidade do município e os recursos existentes. Esse fato já era esperado visto que a realidade das cidades brasileiras é diversa e os recursos não são distribuídos de maneira igualitária. Assim, fica claro que o planejamento municipal com base no entendimento da realidade local se torna de suma importância quando se almeja uma implementação efetiva de alguma política pública, sobretudo quando se fala na cessação do uso do tabaco.

\section{Conclusão}

É notável a importância do PTT do MS para a saúde e melhoria na qualidade de vida dos tabagistas, uma vez que ao controlarem o uso do tabaco passam a obter melhores desfechos no tratamento clínico de suas comorbidades e deixam de apresentar os sintomas e alterações corporais decorrentes do vício. Além disso, também fica claro que o PTT se mostra de suma importância no que tange ao processo redução de gastos financeiros do sistema de saúde, visto que o programa atua diretamente na prevenção das doenças evitáveis que são provocadas pelo tabaco.

O programa no município alvo desta pesquisa apresentou eficácia em promover a cessação do tabagismo, porém com uma porcentagem de sucesso que poderia ser mais satisfatória desde que preenchidas determinadas lacunas e tomadas as medidas cabíveis para a melhoria de sua implementação. Nesse sentido, considerando os resultados atingidos pelo município alvo e as discussões presentes na literatura científica, fica evidente que, para se chegar a desfechos satisfatórios na implementação do PTT, a consideração da realidade econômica e sociodemográfica de cada município ganha importante destaque.

Esse estudo apresentou como limitação o fato de ser transversal e, portanto, não se beneficiar das vantagens de um estudo longitudinal representadas, entre outros fatores, pela possibilidade de tomar notas das mudanças, fazer observações ao longo do processo de tratamento e detectar quaisquer alterações que, porventura, possam ocorrer nas características de seus participantes.

Por fim, observa-se que há uma clara necessidade deste tema ser mais aprofundado, mais investigado e mais discutido para que possam ser criadas estratégias para uma prevenção e tratamento progressivamente efetivos. Portanto, são necessários mais estudos que se proponham a comparar diversas metodologias para cessação do tabagismo e que investiguem estratégias de prevenção do tabagismo na saúde pública e o emprego de novas tecnologias psicoterapêuticas e farmacológicas para que se aumentem as taxas de sucesso.

\section{Referências}

Aragão, E. I. S., Portugal, F. B., Campos, M. R., Lopes, C. D. S., \& Fortes, S. L. C. L. (2017). Distintos padrões de apoio social percebido e sua associação com doenças físicas (hipertensão, diabetes) ou mentais no contexto da atenção primária. Ciência \& Saúde Coletiva, 22, $2367-2374$.

Azevedo, R. C. S. de., Higa, C. M. H., Assumpção, I. S. M. A. de., Frazatto, C. R. G., Fernandes, R. F., Goulart, W., Botega, N. J., Boscolo, M. M., \& Sartori, R. M. (2009). Grupo terapêutico para tabagistas: resultados após seguimento de dois anos. Revista da Associação Médica Brasileira, 55(5), 593-596.

Barbosa, L. F. M., \& Machado, C. J. (2015). Fatores socioeconômicos e culturais associados à prevalência de tabagismo entre trabalhadores do Sistema Único de Saúde em Belo Horizonte. Revista Brasileira de Epidemiologia, 18, 385-397. 
Bazotti, A., Finokiet, M., Conti, I. L., França, M. T. A., \& Waquil, P. D. (2016). Tabagismo e pobreza no Brasil: uma análise do perfil da população tabagista a partir da POF 2008-2009. Ciência \& Saúde Coletiva, 21, 45-52.

Brasil. Ministério da Educação. Gabinete do Ministro. (2013). Portaria n ${ }^{\circ}$ 571, de 5 de abril de 2013. Atualiza as diretrizes de cuidado à pessoa tabagista no âmbito da Rede de Atenção à Saúde das Pessoas com Doenças Crônicas do Sistema Único de Saúde (SUS) e dá outras providências. Diário Oficial da União, Brasília, DF.

Brasil. Ministério da Saúde. (2015). Estratégias para o cuidado da pessoa com doença crônica, o cuidado da pessoa tabagista. Cadernos de Atenção Básica, n. 40. Brasília, DF, p. 55-60.

Brasil. Ministério da Saúde. Secretaria de Atenção à Saúde. (2016). Portaria No 761, de 21 de junho de 2016. Valida as orientações técnicas do tratamento do tabagismo constantes no Protocolo Clínico e Diretrizes Terapêuticas - Dependência à Nicotina. Diário Oficial da União, Brasília, DF.

Brasil. Ministério da Saúde. Instituto Nacional de Câncer José Alencar Gomes da Silva (INCA). (2019a). Deixando de Fumar sem Mistérios: Manual do Coordenador (2a ed.). Rio de Janeiro: INCA.

Brasil. Ministério da Saúde. Instituto Nacional de Câncer José Alencar Gomes da Silva (INCA). (2019b). Tabaco e Saúde Pulmonar: Dia Mundial Sem Tabaco (Manual 2019). Rio de Janeiro: INCA.

Brasil. Ministério da Saúde. Secretaria de Vigilância em Saúde. Departamento de Análise em Saúde e Vigilância de Doenças Não Transmissíveis. (2020a). Vigilância de fatores de risco e proteção para doenças crônicas por inquérito telefônico (VIGITEL): Estimativas sobre frequência e distribuição sociodemográfica de fatores de risco e proteção para doenças crônicas nas capitais dos 26 estados brasileiros e no Distrito Federal em 2019. Brasília, DF.

Brasil. Subsecretaria de Vigilância à Saúde. Secretaria de Saúde do Distrito Federal. (2020b) Boletim Epidemiológico do Tabagismo no Distrito Federal (ano $\left.02, \mathrm{n}^{\circ} 01\right)$. Brasília, DF.

Carlotto, I. N., \& Dinis, M. A. P. (2018). Tecnologias da Informação e Comunicação (TICs) na Promoção da Saúde: Considerações Bioéticas: Information and Communication Technologies (ICTs) in the health promotion: Bioethics considerations. Saber \& Educar, (25), 1-10.

Costa-Júnior, F. M. D., Couto, M. T., \& Maia, A. C. B. (2016). Gênero e cuidados em saúde: Concepções de profissionais que atuam no contexto ambulatorial e hospitalar. Sexualidad, Salud y Sociedad (Rio de Janeiro), (23), 97-117.

Donny, E. C., Denlinger, R. L., Tidey, J. W., Koopmeiners, J. S., Benowitz, N. L., Vandrey, R. G., ... \& Hatsukami, D. K. (2015). Randomized trial of reduced-nicotine standards for cigarettes. New England Journal of Medicine, 373(14), 1340-1349.

França, S. A. D. S., Neves, A. L. F. D., Souza, T. A. S. D., Martins, N. C. N., Carneiro, S. R., Sarges, E. D. S. N. F., \& Souza, M. D. F. A. H. D. (2015). Fatores associados à cessação do tabagismo. Revista de Saúde Pública, 49, 10.

Goyatá, S. L. T., Silva, M. J. D., Souza, W. A. de, Podestá, M. H. M. C., \& Beijo, L. A. (2014). Impacto do programa de apoio ao tabagista de um município do Sul de Minas Gerais, Brasil. Ciencia y Enfermería, 20(1), 75-88.

Higgins, S. T., Bergeria, C. L., Davis, D. R., Streck, J. M., Villanti, A. C., Hughes, J. R., \& Miller, M. E. (2018). Response to reduced nicotine content cigarettes among smokers differing in tobacco dependence severity. Preventive medicine, 117, 15-23.

Lucchese, R., Vargas, L. S., Teodoro, W. R., Santana, L. K. B., \& Santana, F. R. (2013). A tecnologia de grupo operativo aplicada num programa de controle do tabagismo. Texto \& Contexto-Enfermagem, 22(4), 918-926.

Meier, D. A. P., Vannuchi, M. T. O., \& Oliveira Secco, I. A. de (2012). Abandono do tratamento do tabagismo em programa de município do norte do Paraná. Espaço para Saúde, 13(1), 35-44.

Mendes, A. C. R., Toscano, C. M., Barcellos, R. M. D. S., Ribeiro, A. L. P., Ritzel, J. B., Cunha, V. D. S., \& Duncan, B. B. (2016). Costs of the smoking cessation program in Brazil. Revista de saude publica, 50, 66.

Paiva, M. R. A. B. de, Souza, W. A. de, Goyatá, S. L. T., de Siqueira, L. M., Jr, Podestá, M. H. M. C., \& Ferreira, E. B. (2017). Grupo de apoio ao tabagista na estratégia de saúde da família: fatores de sucesso. Revista da Universidade Vale do Rio Verde, 15(2), 436-448.

Pereira, A. S., Shitsuka, D. M., Parreira, F. J., \& Shitsuka, R. (2018). Metodologia da pesquisa científica. UFSM. https://repositorio.ufsm.br/bitstream/handle/1/15824/Lic_Computacao_Metodologia-Pesquisa-Cientifica.pdf?sequence=1.

Pinto, A. C. S., Scopacasa, L. F., Bezerra, L. L. D. A. L., Pedrosa, J. V., \& Pinheiro, P. N. D. C. (2017). Uso de tecnologias da informação e comunicação na educação em saúde de adolescentes: revisão integrativa. Rev. enferm. UFPE on line, 634-644.

Polosa, R., Caponnetto, P., Niaura, R., \& Abrams, D. (2017). Analysis of E-cigarette use in the 2014 Eurobarometer survey: calling out deficiencies in epidemiology methods. Internal and emergency medicine, 12(6), 733-735.

Santana, A. D. M. de, Vasconcellos, L. S. de, \& Ribeiro, M. P. (2017). Grupo de tabagismo: uma abordagem interdisciplinar. Cadernos de Educação, Saúde e Fisioterapia, 4(8).

Santos, M. D. D. V., Santos, S. V., \& Caccia-Bava, M. D. C. G. G. (2019). Prevalência de estratégias para cessação do uso do tabaco na Atenção Primária à Saúde: uma revisão integrativa. Ciência \& Saúde Coletiva, 24, 563-572.

Souza, I. W. de, Kozasa, E. H., Rabello, L. A., Mattozo, B., Bowen, S., Richter, K. P., Sartes, L. M. A., \& Noto, A. R. (2019). Dispositional mindfulness, affect and tobacco dependence among treatment naive cigarette smokers in Brazil. Tobacco induced diseases, 17.

Souza, M. C. D., Cruz, O. G., \& Vasconcelos, A. G. G. (2016). Fatores associados à sobrevida doença-específica em pacientes com câncer de pulmão de células não pequenas. Jornal Brasileiro de Pneumologia, 42(5), 317-325. 
Research, Society and Development, v. 10, n. 2, e47310212695, 2021

(CC BY 4.0) | ISSN 2525-3409 | DOI: http://dx.doi.org/10.33448/rsd-v10i2.12695

Vargas, L. S., Lucchese, R., Silva, A. C. D., Guimarães, R. A., Vera, I., \& Castro, P. A. D. (2017). Determinants of tobacco use by students. Revista de saude publica, 51, 36 .

Viacava, F., Oliveira, R. A. D. D., Carvalho, C. D. C., Laguardia, J., \& Bellido, J. G. (2018). SUS: oferta, acesso e utilização de serviços de saúde nos últimos 30 anos. Ciência \& saúde coletiva, 23, 1751-1762.

World Health Organization. (2019). WHO global report on trends in prevalence of tobacco smoking 2000-2025, third edition. Geneva: World Health Organization. 